Sir,

\section{Endophthalmitis following cataract surgery}

I read with great interest the article by Kamalarajah $\mathrm{et} \mathrm{al}^{1}$ on 'Surveillance of endophthalmitis following cataract surgery in the UK'. The authors deserve to be congratulated for the good effort. There are certain points that I would like to mention to supplement their observations.

1. The importance of formation of a national registry for endophthalmitis is increasingly being recognized in India, as well. ${ }^{2}$ The factors hampering this in our country are the presence of only a few institutes with a established protocol and the excess of private practitioners that prevent the introduction of uniformity in management and reporting. Such a registry would help in establishment of a standard of care in endophthalmitis as well as fuel research on ideal region-specific antimicrobial agents.

2. Although the role of Diabetes mellitus in the occurrence of postoperative infectious endophthalmitis (PIE) is not clear at present, its incidence ought to have been mentioned in this study. In a series from India, diabetic eyes with PIE had a significantly worse outcome than nondiabetic eyes. ${ }^{3}$ The endophthalmitisvitrectomy study (EVS) too found a difference between diabetic and nondiabetic eyes with PIE in the form of a higher rate of culture-positivity in the former. ${ }^{4}$

3. It is highly surprising that a virulent organism such as Pseudomonas was cultured from the anterior chamber aspirate and not the vitreous in three cases! The anterior chamber has a better ability to contain infection than the vitreous, mainly due to the phagocytic capability of the iris endothelial cells. ${ }^{5}$ Hence, culture-positivity from an aqueous aspirate denotes overwhelming infection and the vitreous sample must test positive as well. Although the authors have quoted similar observations in another publication, the possibility of contamination from the conjunctival flora during sample collection or in the lab during sample processing must be ruled out.

4. It is indeed significant that in this series of cases from entire UK, there was not a single case of PIE due to fungi! Another study from the UK by Heaven et al, ${ }^{6}$ too reported a nil incidence of fungal PIE. This however, is not the Indian experience, where fungi are being increasingly isolated even in cases of acute PIE. ${ }^{3,7,8}$ Although it is possible that this difference could be attributed to tropical climate, factors such as differences in inclusion criteria or random variation could also play a role. Knowledge of the susceptibility of the ocular isolates in this series would also be of great interest to see for any similar geographic variation in microbial resistance.
5. The authors have noted that despite the EVS providing evidence of the lack of benefit of systemic antibiotics in PIE, $65 \%$ of the patients in the series did receive them. In this context, I would like to point out that the EVS did not test the efficacy of antibiotics such as fluoroquinolones that have a good intravitreal penetration. ${ }^{9}$ We do administer systemic antibiotics such as oral ciprofloxacin to the PIE cases at our institute. This may be expected to have a synergistic effect with the intravitreal antibiotic such as vancomycin, which would be all the more important considering that there is no assistance from the body's immune system in combating intraocular infection. ${ }^{10}$

\section{Acknowledgements}

Financial interest: None.

\section{References}

1 Kamalarajah S, Silvestri G, Sharma N, Khan A, Foot B, Ling $\mathrm{R}$ et al. Surveillance of endophthalmitis following cataract surgery in the UK. Eye 2004; 18: 580-587.

2 Das T. National endophthalmitis survey. Ind J Ophthalmol 2003; 51: 117-118.

3 Gupta A, Gupta V, Gupta A, Dogra MR, Pandav SS, Ray P et al. Spectrum and clinical profile of postcataract surgery endophthalmitis in north India. Ind J Ophthalmol 2003; 51: 139-145.

4 Johnson MW, Doft BH, Kelsey SF, Barza M, Wilson LA, Barr $\mathrm{CC}$ et al. The endophthalmitis vitrectomy study. Relationship between clinical presentation and microbiologic spectrum. Ophthalmology 1997; 104: 261-272.

5 Maylath FR, Leopald IH. Study of experimental intraocular infection. Am J Ophthalmol 1955; 40: 86-101.

6 Heaven CJ, Mann PJ, Boase DL. Endophthalmitis following extracapsular cataract surgery. Br J Ophthalmol 1992; 76: 419-423.

7 Anand AR, Therese KL, Madhavan HN. Spectrum of aetiological agents of postoperative endophthalmitis and antibiotic susceptibility of bacterial isolates. Indian J Ophthalmol 2000; 48: 123-128.

8 Kunimoto DY, Das T, Sharma S, Jalali S, Majji AB, Gopinathan $\mathrm{U}$ et al and the Endophthalmitis Research Group. Microbiologic spectrum and susceptibility of isolates: Part I. Post operative endophthalmitis. Am J Ophthalmol 1999; 128: 240-242.

9 Keren G, Alhalel A, Bartov E, Kitzes-Cohen R, Rubinstein E, Segey $\mathrm{S}$ et al. The intravitreal penetration of orally administered ciprofloxacin in humans. Invest Ophthalmol Vis Sci 1991; 32: 2388-2392.

10 Vedantham V. Ciprofloxacin in endophthalmitis: an emerging alternative. Am J Ophthalmol 2004; 137: 1167-1168.

\section{Vedantham}

Retina-Vitreous service

Aravind Eye Hospital \& PG Institute of Ophthalmology

1 Kurivikaran Salai

Anna Nagar 
Madurai

Tamilnadu 625020, India

Correspondence: $\vee$ Vedantham

Tel: + 914525356100

Fax: + 914522530984

E-mail: drvasumathy@yahoo.com

Eye (2005) 19, 1219-1220. doi:10.1038/sj.eye.6701723; published online 1 October 2004

Sir,

\section{Reply to V Vedantham}

We thank Vasumathy Vedantham for his interest in our paper. In reply to his comments:

1. The British Ophthalmological Surveillance Unit (BOSU) is now well established in the UK to assist in the investigation of clinically important or rare eye conditions such as endophthalmitis. The BOSU-reporting scheme is dependent on voluntary reporting and therefore under-reporting of cases is a potential source of error for incidence calculations. A national registry is ideal for monitoring of postoperative endophthalmitis cases but would require the support of the vast majority of ophthalmologists in the UK or India.

2. The incidence of diabetes in the endophthalmitis cases was $10 \%$. This figure was mentioned in our paper under the heading of cataract surgery details. Comparison of outcome between diabetic and nondiabetic eyes was not analysed in this paper.

3. Pseudomonas was cultured from the aqueous sample but not the vitreous in 3/5 Pseudomonas isolates. This was a surprising result as vitreous is a better culture medium for bacteria than aqueous; ${ }^{1}$ however, cases with a positive aqueous tap and negative vitreous tap have been described. ${ }^{1-2}$

As this study was dependent on individual UK centres providing specific information about endophthalmitis cases, we cannot comment on the vitreous/aqueous sampling technique or sample processing at these centres.

4. In contrast to several Indian endophthalmitis series, ${ }^{3-5}$ no cases of fungi were isolated in our UK survey. Fungal endophthalmitis following cataract surgery has been described as having a prolonged latency period of weeks to months after intraocular inoculation., 5 For our UK survey, we only included cases diagnosed within 6 weeks of cataract surgery, excluding potential delayed onset cases due to fungal infection.
Differences in climate, operating theatre conditions or the sample processing technique may also be important. Information on the antibiotic susceptibility of ocular isolates was not requested for our study.

5. The role of systemic antibiotics in the treatment of endophthalmitis remains unclear. Patients randomised to the intravenous (IV) antibiotic group in the Endophthalmitis Vitrectomy Study (EVS) ${ }^{7}$ received ceftazidime and amikacin (oral ciprofloxacin instead of ceftazidime if the patient was allergic to penicillin). There was no statistically significant difference in outcomes between the IV antibiotic group and the control group. Animal studies have shown inadequate intraocular drug levels of amikacin in inflamed, aphakic, vitrectomized eyes following IV treatment. ${ }^{8}$ IV vancomycin or cefazolin may provide better Gram-positive coverage in aphakic, inflamed eyes than ceftazidime or amikacin..$^{9}$ Therefore, the results of systemic antibiotics in the EVS may not apply to other drug combinations.

\section{References}

1 Beyer TL, Vogler G, Sharma D, O'Donnell FE. Protective barrier effect of the posterior lens capsule in exogenous bacterial endophthalmitis - an experimental primate study. Invest Ophthalmol Vis Sci 1984; 25: 108-112.

2 Barza M, Pavan PR, Doft BH, Wisniewski SR, Wilson LA, Han $\mathrm{DP}$ et al. Evaluation of microbiological diagnostic techniques in postoperative endophthalmitis in the Endophthalmitis Vitrectomy Study. Arch Ophthalmol 1997; 115(9): 1142-1150.

3 Anand AR, Therese KL, Madhavan HN. Spectrum of aetiological agents of postoperative endophthalmitis and antibiotic susceptibility of bacterial isolates. Indian J Ophthalmol 2000; 48: 123-128.

4 Kunimoto DY, Das T, Sharma S, Jalali S, Majji AB, Gopinathan $U$ et al. Microbiologic spectrum and susceptibility of isolates: part I. Postoperative endophthalmitis. Endophthalmitis Research Group. Am J Ophthalmol 1999; 128(2): 240-242.

5 Narang S, Gupta A, Gupta V, Dogra MR, Ram J, Pandav SS et al. Fungal endophthalmitis following cataract surgery: clinical presentation, microbiological spectrum and outcome. Am J Ophthalmol 2001; 132: 609-617.

6 Pflugfelder SC, Flynn HW, Zwickey TA, Forster RK, Tsiligianni A, Culbertson W et al. Exogenous fungal endophthalmitis. Ophthalmology 1988; 95: 19-30.

7 EVS. Results of the Endophthalmitis Vitrectomy Study. A randomized trial of immediate vitrectomy and of intravenous antibiotics for the treatment of postoperative bacterial endophthalmitis. Endophthalmitis Vitrectomy Study Group. Arch Ophthalmol 1995; 113(12): 1479-1496.

8 El-Massry A, Meredith TA, Aguilar HE, Shaarawy A, Kincaid $\mathrm{M}$, Dick J et al. Aminoglycoside levels in the rabbit vitreous cavity after intravenous administration. Am J Ophthalmol 1996; 122: 684-689.

9 Davis JL. Intravenous antibiotics for endophthalmitis. Am J Ophthalmol 1996; 122(5): 724-726. 